\title{
Anomalous Small-Angle X-Ray Scattering Characterization of Composites Based on Sulfonated Poly(ether ether ketone), Zirconium Phosphates, and Zirconium Oxide
}

\author{
L. A. S. DE A. PRADO, ${ }^{1}$ H. WITTICH, ${ }^{1}$ K. SCHUlte, ${ }^{1}$ G. GOERIGK, ${ }^{2}$ VASIL M. GARAMUS, ${ }^{3}$ R. WILlUMEIT, ${ }^{3}$ \\ S. VETTER, ${ }^{3}$ B. RUFFMANN, ${ }^{3}$ S. P. NUNES ${ }^{3}$ \\ ${ }^{1}$ Polymer Composites, TU Hamburg-Harburg, Denickerstrasse 15, D-21073 Hamburg-Harburg, Germany \\ ${ }^{2}$ Institute of Solid State Research, Jülich Research Centre, P.O. Box 1913, D-52425 Jülich, Germany \\ ${ }^{3}$ GKSS Research Centre, Max-Planck Strasse 1, D-21520 Geesthacht, Germany
}

Received 20 June 2003; revised 29 September 2003; accepted 7 October 2003

\begin{abstract}
The morphology and distribution of zirconium oxide and zirconium phosphates in a matrix of sulfonated poly(ether ether ketone) (SPEEK) were investigated with anomalous small-angle X-ray scattering (ASAXS) and electron microscopy. ASAXS revealed that $\mathrm{ZrO}_{2}$ was distributed in the SPEEK matrix in the form of nanoparticles smaller than $13 \AA$. A decrease in the conductivity suggested that the sulfonic groups were bound to the zirconium oxo species at the particle surface. Furthermore, two kinds of membranes containing zirconium phosphate were investigated. In one case, the phosphate was directly dispersed in the polymer solution for the casting of the membrane. In the other case, the phosphate was previously treated with $n$-propyl ammonium and polybenzimidazole. From ASAXS data, the fractal dimension could be estimated. Mass-fractal behavior was confirmed for the SPEEK membrane containing previously exfoliated zirconium phosphate, with aggregates of 6.3-165 A. Surfacefractal behavior was detected for membranes with untreated phosphates, with aggregates of 6.4-185 A. The untreated phosphates caused an increase in the permeability, without changing the proton conductivity much. () 2003 Wiley Periodicals, Inc. J Polym Sci Part B: Polym Phys 42: 567-575, 2003
\end{abstract}

Keywords: anomalous small-angle X-ray scattering; composites; membranes; microstructure

\section{INTRODUCTION}

Organic-inorganic hybrid materials are under fast development. ${ }^{1}$ Alkoxide precursors can be adequately functionalized to improve the compatibility between organic and inorganic phases, either by chemical bonding ${ }^{2,3}$ or by intermolecular forces, such as hydrogen bonding. ${ }^{4}$ In hybrids, the

Correspondence to: S. P. Nunes (E-mail: nunes@gkss.de) Journal of Polymer Science: Part B: Polymer Physics, Vol. 42, 567-575 (2003) ๑) 2003 Wiley Periodicals, Inc. thermal stability, mechanical properties, and swelling behavior of polymers can be tailored to meet the requirements for different applications, such as insulating films, sensors, and optical, magnetic, and electric devices. For membrane applications, the introduction of a finely dispersed inorganic phase has been demonstrated to be successful in controlling selectivity and permeability for gas separation, ${ }^{5}$ decreasing compaction, ${ }^{6}$ and swelling. ${ }^{7}$ More recently, the same composites investigated in this study have been used as membrane material for direct methanol fuel cells. ${ }^{8}$ 
Zirconium oxide has been used to reduce methanol and water crossover in membranes. However, zirconium phosphate has been used to help with proton transport. ${ }^{8}$

In this study, anomalous small-angle X-ray scattering (ASAXS) was applied to investigate the distribution of zirconium oxide and phosphates subjected to different treatments in a matrix of sulfonated poly(ether ether ketone) (SPEEK).

\section{ASAXS}

Scattering methods have already been established as characterization tools for membranes, ${ }^{9,10}$ polymer networks, ${ }^{11,12}$ and polymeric materials, including ionomers. In small-angle Xray scattering (SAXS) experiments, fluctuations of the electron density give rise to characteristic patterns due to the scattering of incident X-rays. The scattering intensity $[I(\mathbf{q})]$ can be plotted against the scattering vector $(\mathbf{q})$ :

$$
\mathbf{q}=\frac{4 \pi}{\lambda} \sin \left(\frac{\theta}{2}\right)
$$

where $\theta$ and $\lambda$ are the scattering angle and wavelength of the incident X-rays, respectively.

With synchrotron radiation, the energy of the incident beam can be tuned to enable SAXS measurements in the immediate neighborhood of the $\mathrm{X}$-ray absorption edges of the heavier elements present in a sample. This element-specific technique is known as ASAXS and uses the dependence of the atomic scattering factor $[f(E)]$ on the energy of the incident X-ray $(E)$ :

$$
f(\mathbf{q} E)=f^{0}(\mathbf{q})+f^{\prime}(\mathbf{q}, E)+i f^{\prime}(E, \mathbf{q})
$$

The nonresonant factor $\left[f^{0}(\mathbf{q})\right]$ is energy-independent and gives the number of electrons of the element. The so-called anomalous dispersion corrections $\left[f^{\prime}(\mathbf{q}, E)\right.$ and $\left.f^{\prime}(\mathbf{q}, E)\right]$ are energy-dependent and undergo drastic changes in the vicinity of the X-ray absorption edges. The scattering due to the chemical element under investigation can be separated by the subtraction of two (or more) scattering curves measured at energies near the absorption edge.

A recent review of ASAXS applications for the characterization of polymer colloids was published by Ballauff, ${ }^{13}$ and it has also been applied to other polymer systems containing metals. ${ }^{14-16}$ In this field, Cooper and coworkers ${ }^{17-19}$ made an important contribution, studying the ASAXS curves of ionomers neutralized with heavy metals. Here we focus on the investigation of the distribution of $\mathrm{Zr}$-containing compounds in a matrix of SPEEK.

\section{Fractal Dimension}

A common approach is the analysis of the power law of $I(\mathbf{q})$, which allows the determination, for instance, of the fractal dimension. The intensity in the power-law region can be described as follows: ${ }^{20-22}$

$$
I(\mathbf{q}) \propto \mathbf{q}^{-\alpha}
$$

The angular coefficient $(\alpha)$ of the plot of $\log I(\mathbf{q})$ versus log $\mathbf{q}$ can be determined from the experimental SAXS pattern, and it is correlated to the mass-fractal dimension $\left(D_{\mathrm{v}}\right)$ and to the surfacefractal dimension $\left(D_{\mathrm{s}}\right):^{23}$

$$
\alpha=2 D_{\mathrm{v}}-D_{\mathrm{s}}
$$

$\alpha$ also determines whether the scattering units are bound in a surface fractal or in a mass fractal. Mass fractals correspond to open structures, whereas surface fractals describe dense materials with rough surfaces. If $1<\alpha<3$, the curve corresponds to a mass-fractal material. In these systems, often called volume or bulky fractals, the surface and the bulk are not uniformly dense; that is, the surface and the inner part of the structure have the same fractal dimension, and so $D_{\mathrm{v}}$ is equal to $D_{\mathrm{s}}$.

However, if $3<\alpha<4$, the material has a dense core $\left(D_{\mathrm{v}}=3\right)$, and $D_{\mathrm{s}}$ is equal to $6-\alpha$, which corresponds to surface-fractal behavior. For a nonfractal material, $D_{\mathrm{v}}$ is 3 and $D_{\mathrm{s}}$ is 2 (a uniformly dense and perfectly smooth surface); $\alpha$ is $4 .^{23}$

\section{Models for Ionomers ${ }^{24}$}

In the field of ionomers, SAXS curves and, less frequently, ASAXS curves have been analyzed according to different models. For instance, in Nafion or acrylate-based ionomers, in which there is a strong aggregation of the ionic groups, giving rise to clusters and multiplets, a peak, termed ionomer peak, is present in the curves, as well as an upturn at low-angle values. Actually, there is no satisfactory model to fit the peak and the upturn of the SAXS curves. The existing models 
were reviewed and discussed elsewhere by Gebel and Loppinet ${ }^{24}$ and can be divided into three kinds: the isolated cluster model, the core-shell model, and the radial distribution.

\section{EXPERIMENTAL}

\section{Materials}

Zirconium tetrapropylate was supplied as a 70\% solution in 1-propanol (Aldrich); zirconium oxychloride octahydrate $\left(\mathrm{ZrOCl}_{2} \cdot 8 \mathrm{H}_{2} \mathrm{O}\right)$ and acetylacetone were provided by Fluka and were used without previous purification. $N$-Methylpyrrolidone (NMP) was used as a solvent for the membrane casting process.

SPEEK was obtained in the laboratory by the sulfonation of poly(ether ether ketone) $450 \mathrm{P}$, supplied in pellets by Victrex, with a procedure described previously. ${ }^{8}$ The conditions were adjusted to reach a sulfonation degree of $40 \%$.

\section{Plain SPEEK Membrane and SPEEK/ZrO ${ }_{2}$ Composites}

Plain SPEEK membranes were prepared from solutions containing $7 \mathrm{wt} \%$ SPEEK in NMP. For the preparation of SPEEK/Z $\mathrm{ZrO}_{2}$ composites, zirconium propylate and acetylacetone (in a molar ratio of ca. 1:3) and small amounts of water (ca. $0.05 \mathrm{~g}$ of water/g of polymer) were added to the solution and stirred for $16 \mathrm{~h}$ at room temperature to complete the hydrolysis and condensation of the alkoxide. The final composite contained 13.5 wt $\% \mathrm{ZrO}_{2}$ in the polymer matrix.

\section{SPEEK/Zirconium Phosphate Composites}

Zirconium phosphate was prepared; the preparation began with the neutralization of a $0.1 \mathrm{M}$ zirconium oxychloride aqueous solution with ammonium hydroxide, under reflux, according to a procedure adapted from that described in the literature. ${ }^{25}$ An aqueous dispersion of zirconium oxide was obtained. The oxide was separated by centrifugation and treated with phosphoric acid in dimethylformamide (DMF). After being washed by repeated centrifugation and redispersion, the resulting zirconium phosphate dispersion in DMF was mixed with the SPEEK solution. After solvent evaporation, a composite containing $23 \%$ zirconium phosphate in the polymer matrix was obtained.
In an alternative procedure, the zirconium phosphate dispersion was reacted with a $1 \mathrm{M}$ $n$-propyl amine solution ( $0.1 \mathrm{~g}$ of $n$-propyl amine/g of zirconium phosphate). The dispersion was further stirred in the presence of polybenzimidazole (PBI; $0.54 \mathrm{~g}$ of PBI/g of zirconium phosphate) in an attempt to exfoliate the layers of zirconium phosphate. After that, the dispersion was mixed with a SPEEK solution in NMP to give composites containing 23 wt \% zirconium phosphate.

\section{Casting of the Membranes}

All the solutions containing SPEEK and the inorganic component were cast onto a glass plate heated to temperatures ranging from 40 to $90{ }^{\circ} \mathrm{C}$ for solvent evaporation. The glass plate was previously hydrophobized with octadecyl trichlorosilane.

\section{Scanning Electron Microscopy (SEM)}

Cross sections of the membranes were observed in a LEO Gemini field emission scanning electron microscope after the membranes were fractured in 2-propanol and coated with $\mathrm{Au} / \mathrm{Pd}$.

\section{Transmission Electron Microscopy}

The samples were cut with diamond knife at about $-40{ }^{\circ} \mathrm{C}$ in a Leica Ultracut UCT ultramicrotome with a Leica EM FCS cryosystem and were observed in a Zeiss EM-902 transmission electron microscope.

\section{Proton Conductivity Measurements}

The proton conductivity was measured with a Zahner IM6 impedance spectrometer with a measuring cell similar to that described by Alberti et al. ${ }^{26}$ The membranes were pressed between Ethek electrodes, and the measurements were performed at $100 \%$ relative humidity from room temperature up to $110{ }^{\circ} \mathrm{C}$.

\section{Pervaporation}

The permeability of methanol and water across the membranes was measured in a pervaporation setup described previously. ${ }^{27}$ A $20 \%$ methanol aqueous solution was used as the feed. The membrane was positioned in a Millipore 47-mm-diameter cell, and the permeate side was kept in vacuo. The permeate was condensed in liquid ni- 


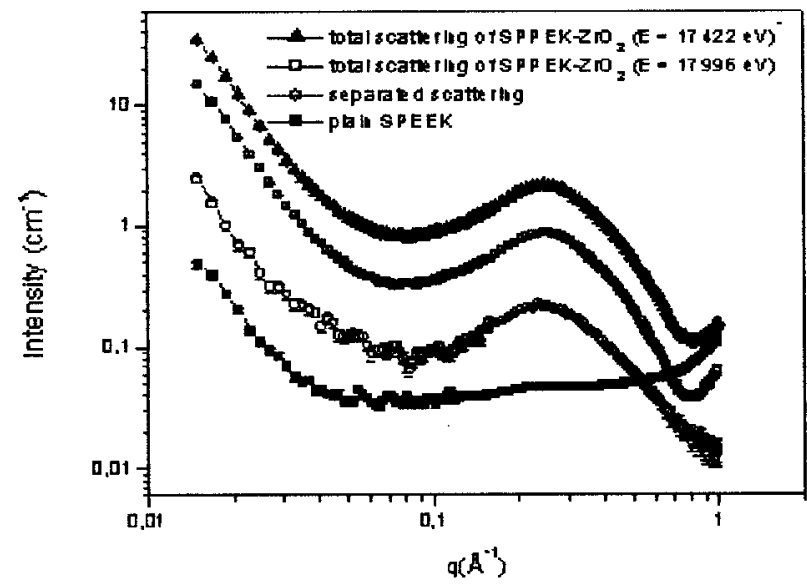

Figure 1. SAXS curves for plain SPEEK and ASAXS plots for SPEEK-Z $\mathrm{rO}_{2}$ membranes, including total and separated scattering curves. The total scattering curve at $E=17422 \mathrm{eV}$ was shifted vertically for clarity.

trogen for $1 \mathrm{~h}$, and the weight and composition were determined by the measurement of the refraction index of the solution.

\section{ASAXS Experiments}

The ASAXS measurements were performed at the JUSIFA beam line at DESY-HASYLAB (Hamburg, Germany) ${ }^{28}$ with different energies below the $\mathrm{Zr}-\mathrm{K}$ absorption edge at $17.998 \mathrm{keV}$ and with two sample-detector distances (935 and 3635 $\mathrm{mm}$ ) to cover the broadest $\mathbf{q}$ range between 0.01 and $1 \AA^{-1}$. The intensities were normalized to the primary beam intensity and corrected for sample transmission, background, and detector efficiency. The scattering curves were calibrated into macroscopic scattering cross sections in units of the cross section per unit of volume $\left(\mathrm{cm}^{2} / \mathrm{cm}^{3}\right.$ $=\mathrm{cm}^{-1}$ ). The two energies of incident X-rays were 17,432 and $17,996 \mathrm{eV}$. The SAXS curve of a plain SPEEK membrane was taken at the lowest energy. Attempts to obtain ASAXS curves with higher energies were hindered by the extensive fluorescence of the samples.

\section{RESULTS AND DISCUSSION}

\section{SAXS of SPEEK Membranes}

Figure 1 shows the SAXS curve of plain SPEEK, that is, without the incorporation of zirconium phosphate (or zirconium oxide), obtained with Xrays with energy equal to $17,432 \mathrm{eV}$. It did not exhibit pronounced peak structures. In other words, the so-called ionomer peak, associated either with phase separation or with clustering of the ionic groups, was not present, and this indicated that the sulfonic groups were randomly distributed in the material. A reason for this is that sulfonic groups are statistically attached to the main chain of poly(ether ether ketone). Furthermore, Guiver et al. ${ }^{29}$ suggested that the rigid nature of the polymer matrix would preclude clustering and further phase-separation processes. A quite different case is observed for polymers such as Nafion, in which the sulfonic group is attached to the polymer side chain; this makes the clustering of ionic groups easier. Also, in the case of block copolymers, the separation of ionic groups in microdomains is more favored than in the SPEEK investigated here.

\section{SPEEK/ZrO ${ }_{2}$ Composites}

The in situ generation of $\mathrm{ZrO}_{2}$ nanoparticles in SPEEK solutions was a useful procedure for preparing membranes with low methanol and water permeability, as reported before. ${ }^{8}$ The effect of $\mathrm{ZrO}_{2}$ on the permeability of the membrane can be followed in Table 1. With the generation of 13.5 wt $\% \mathrm{ZrO}_{2}$ in the SPEEK membrane, the total flow of a methanol/water solution across the membrane decreased to $11 \%$ of the value across the plain membrane.

The formation of zirconium oxide occurred via the hydrolysis of the zirconium propylate, starting with the cleavage of $\mathrm{ZrOC}_{3} \mathrm{H}_{7}$ bonds. Because of the high reactivity of this precursor, acetylacetone had to be added to the solution to reduce the polymerization rate. This step was mandatory to produce homogeneous casting solutions and homogeneous membranes therefrom. ${ }^{8}$ The $\mathrm{Zr}-\mathrm{OH}$ bonds reacted further, giving rise to $\mathrm{Zr}-\mathrm{O}-\mathrm{Zr}$ bonds, which were stable to hydrolytic cleavage. The reactions can be summarized as follows:

$$
\begin{aligned}
& \equiv \mathrm{Zr}-\mathrm{OC}_{3} \mathrm{H}_{7}+\mathrm{acacH} \rightarrow \equiv \mathrm{Zr}-\text { acac } \\
& +\mathrm{H}-\mathrm{OC}_{3} \mathrm{H}_{7} \quad \text { (complexation) } \\
& \equiv \mathrm{Zr}-\mathrm{OC}_{3} \mathrm{H}_{7}+\mathrm{H}_{2} \mathrm{O} \rightarrow \equiv \mathrm{Zr}-\mathrm{OH} \\
& +\mathrm{H}-\mathrm{OC}_{3} \mathrm{H}_{7} \quad \text { (hydrolysis) } \\
& \equiv \mathrm{Zr}-\mathrm{OH}+\equiv \mathrm{Zr}-\mathrm{OC}_{3} \mathrm{H}_{7} \rightarrow \equiv \mathrm{Zr}-\mathrm{O}-\mathrm{Zr} \equiv \\
& +\mathrm{H}-\mathrm{OC}_{3} \mathrm{H}_{7} \quad \text { (condensation) }
\end{aligned}
$$


Table 1. Proton Conductivity at $110{ }^{\circ} \mathrm{C}$ and $100 \%$ Relative Humidity and Flux of Water and Methanol across the Membranes at $55{ }^{\circ} \mathrm{C}$ in Pervaporation Experiments with a $20 \%$ Methanol Feed Solution, 1 atm of Pressure in the Feed Side, and a Vacuum in the Permeate Side

\begin{tabular}{lcccc}
\hline & & & \multicolumn{2}{c}{ Pervaporation } \\
\cline { 4 - 5 } Membrane Composition & $\begin{array}{c}\text { Thickness } \\
(\mu \mathrm{m})\end{array}$ & $\begin{array}{c}\text { Conductivity } \\
(\mathrm{mS} / \mathrm{cm})\end{array}$ & $\begin{array}{c}\text { Total Flux } \\
\left(\text { Water }+ \text { Methanol, g/h m }{ }^{2}\right)\end{array}$ & $\begin{array}{c}\text { Methanol in the } \\
\text { Permeate }(\%)\end{array}$ \\
\hline SPEEK & 75 & 3.6 & 922 & 6.5 \\
SPEEK/13.5 wt \% ZrO $\mathrm{Zr}_{2}$ & 103 & 0.003 & 101 & 6.5 \\
SPEEK/23 wt \% phosphate & 76 & 2.3 & 4296 & 9 \\
SPEEK/23 wt \% pretreated & 96 & 0.82 & 44 & 8.5 \\
phosphate & & & & \\
\hline
\end{tabular}

$$
\begin{aligned}
& \equiv \mathrm{Zr}-\mathrm{OH}+\equiv \mathrm{Zr}-\mathrm{OH} \rightarrow \equiv \mathrm{Zr}-\mathrm{O}-\mathrm{Zr} \equiv \\
&+\mathrm{H}-\mathrm{OH} \quad \text { (condensation) }
\end{aligned}
$$

The SPEEK- $\mathrm{ZrO}_{2}$ membranes were cast from homogeneous solutions, and very small domains of the inorganic phase were obtained. This good compatibility between the inorganic phase and polymer could be explained in terms of $\equiv \mathrm{Zr}-\mathrm{O}-\mathrm{SO}_{2} \mathrm{R}$ formation during the polycondensation of the zirconium oxo species. ${ }^{30}$ The reactions can be summarized as follows:

$$
\begin{aligned}
& \mathrm{R}-\mathrm{SO}_{3} \mathrm{H}+\equiv \mathrm{Zr}-\mathrm{OH} \rightarrow \equiv \mathrm{Zr}-\mathrm{SO}_{3}-\mathrm{R} \\
&+\mathrm{H}-\mathrm{OH}(\text { complexation) } \\
& \mathrm{R}-\mathrm{SO}_{3} \mathrm{H}+\equiv \mathrm{Zr}-\mathrm{OC}_{3} \mathrm{H}_{7} \rightarrow \equiv \mathrm{Zr}-\mathrm{SO}_{3}- \\
&+\mathrm{H}-\mathrm{OC}_{3} \mathrm{H}_{7} \text { (complexation) }
\end{aligned}
$$

where $\mathrm{R}$ represents the polymer chain.

They not only represent the complexation of the zirconium by the pendant sulfonic groups but also imply the partial deactivation of the protonconduction sites due to the formation of the $\mathrm{Zr}-\mathrm{O}-\mathrm{SO}_{2} \mathrm{R}$ bonds and to the consumption of the proton attached to the sulfonic group. In Table 1 , a drastic reduction in the proton conductivity (from ca. 4 to $0.003 \mathrm{mS} / \mathrm{cm}$ ) at $100{ }^{\circ} \mathrm{C}$ and $100 \%$ relative humidity can be seen with the introduction of $13.5 \mathrm{wt} \% \mathrm{ZrO}_{2}$.

The real distribution of the oxide particles in the membrane could be determined by ASAXS. In contrast to the plain membrane of SPEEK, the SAXS curves for SPEEK-Z $\mathrm{rO}_{2}$ membranes had a peak (Fig. 1), the maximum of which was located at $0.235 \AA^{-1}$. The presence of a peak in the SAXS pattern can be associated with relatively close scattering units with some spatial correlation. ${ }^{31}$ The fluctuation in the electron density could be caused by the presence of crystallites, as for semicrystalline polymers such as poly(amides) ${ }^{32}$ however, the membranes based on sulfonated poly(ether ketone) and zirconium oxide were amorphous, as previously confirmed by X-ray diffraction. ${ }^{8}$ The fluctuation was caused by the distribution of zirconium oxide particles in the polymer matrix. The distance $(d)$ between the $\mathrm{ZrO}_{2}$ particles was estimated by SAXS from the position of the maximum with eq $5:^{33}$

$$
d=\frac{2 \pi}{\mathbf{q}_{\max }}
$$

The value found was $26.7 \AA$. Two limit situations can be proposed. The particles were really close to one another in a percolated amorphous structure, or they might have been as small as possible but present in a very high concentration. In the latter case, the smallest particle could contain just one $\mathrm{Zr}-\mathrm{O}-\mathrm{Zr}$ bond $\left(2.0 \AA^{34}\right)$; in a percolated structure, the mean distance between the particles could be used to estimate their radius (13.3 $\AA$ ). Therefore, we can say that the particle sizes were between 2.0 and $13.3 \AA$ in a polydisperse distribution. This particles size was analogous to that found for other hybrid materials containing $\mathrm{ZrO}_{2}$ particles chemically bound to the polymer matrix. ${ }^{35,36}$

\section{SPEEK/Zirconium Phosphate Composites}

The inorganic domains in the case of SPEEK/ zirconium phosphate composites were much larger than in the case of $\mathrm{ZrO}_{2}$ composites be- 
cause the phosphate was previously prepared in the form of a dispersion before being added to the polymer solution. The introduction of zirconium phosphate to the SPEEK membranes was designed to contribute to the proton conductivity, in contrast to $\mathrm{ZrO}_{2}$. In Table 1, the proton conductivity is shown to have been practically not altered with the introduction of $23 \mathrm{wt} \%$ zirconium phosphate, whereas with a much lower amount of $\mathrm{ZrO}_{2}$, the conductivity was drastically reduced. The methanol and water permeabilities across the membrane were even increased because of the high hydrophilicity of the phosphate. In a previous article ${ }^{8}$ it was shown that only a combination of $\mathrm{ZrO}_{2}$ and zirconium phosphate in the membrane enabled an optimized balance of proton conductivity and methanol and water permeability.

Alberti et al. ${ }^{37}$ prepared different kinds of zirconium phosphates and investigated their proton conductivity mechanism, which was, to a great extent, related to surface conduction. It was, therefore, expected that the exfoliation of the phosphate layers would lead to an increase in the surface and thus in the conductivity. With this objective in mind and also in an attempt to improve the compatibility with SPEEK, we treated the zirconium phosphate with $n$-propyl amine and further with PBI.

With the compatibility taken into consideration, SEM gives an indication of the effect of the $n$-propyl amine/PBI treatment on the dispersion of the phosphate in the SPEEK matrix. Micrographs are given in Figure 2 for SPEEK/phosphate composites with and without treatment.

In the composites without the treatment, phosphate particles formed agglomerates, detached from the polymer matrix. Individual particles had diameter of less than $50 \mathrm{~nm}$, and several particles formed the agglomerate. When the phosphate particles were previously treated with $n$-propyl amine/PBI, they were more embedded in the polymer matrix, and this indicated a stronger interaction. Therefore, the treatment with PBI helped the compatibilization between the inorganic and polymeric phases. A reason for this is the basic character of PBI, which enabled strong interactions with the acid phosphate and with the acid sulfonic groups in the polymer.

Because of the better compatibilization, the phosphate treated with PBI led to membranes with much lower methanol and water permeability than the nontreated phosphates (Table 1). However, the high acid-base interaction of PBI both with the zirconium phosphate and with the
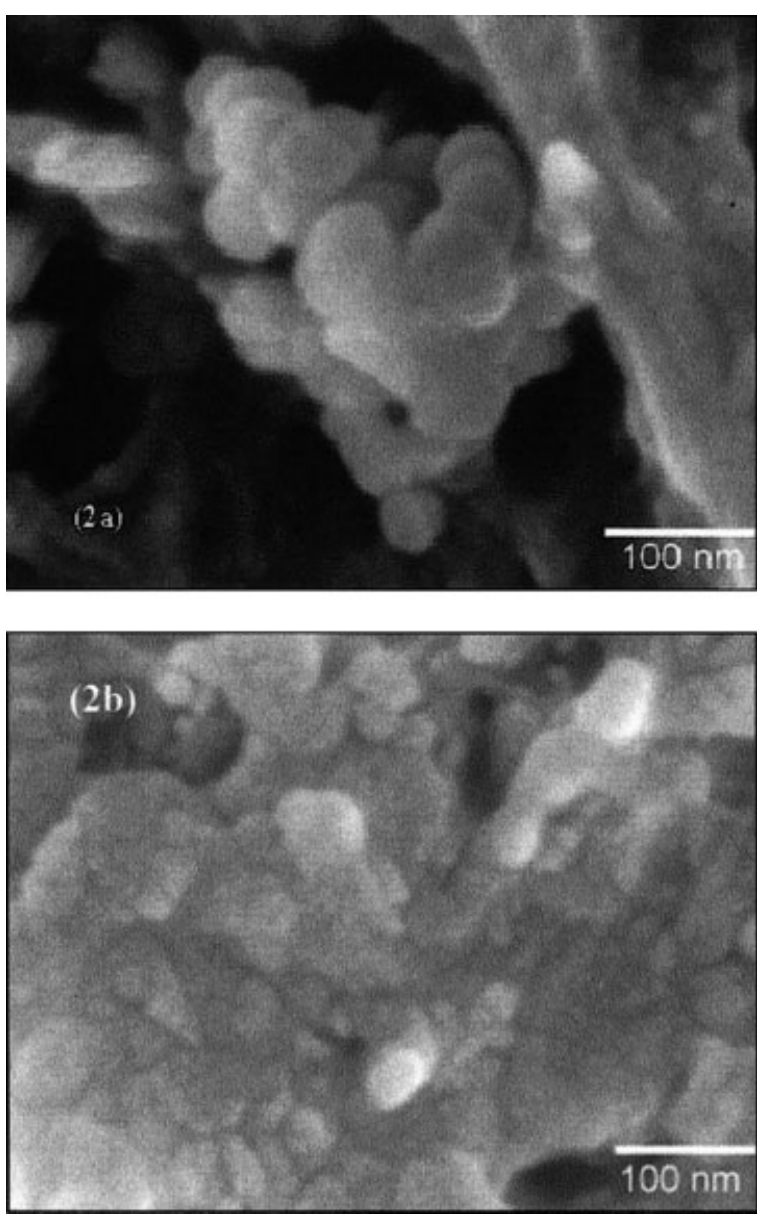

Figure 2. SEM micrographs of SPEEK composites with (a) zirconium phosphates without any pretreatment and (b) zirconium phosphates treated with $n$ propyl amine and PBI.

sulfonic groups of the polymer caused a decrease in the proton conductivity (Table 1) because many of the acid sites responsible for the proton transport were somehow blocked.

Concerning the organization of the phosphate layers themselves in individual particles and their distribution in the polymer composite, transmission electron microscopy was a more informative method. Figure 3 shows a transmission electron microscopy image of a sulfonated poly(ether ketone) composite containing phosphates treated with propylammonium and PBI. Organized phosphate layers can still be observed inside individual particles and are somewhat parallel.

For any composite made from a polymer and a layered material, three possibilities exist: ${ }^{38-40}(1)$ the formation of a microcomposite (i.e., the interlayer distance is not increased after the formation 


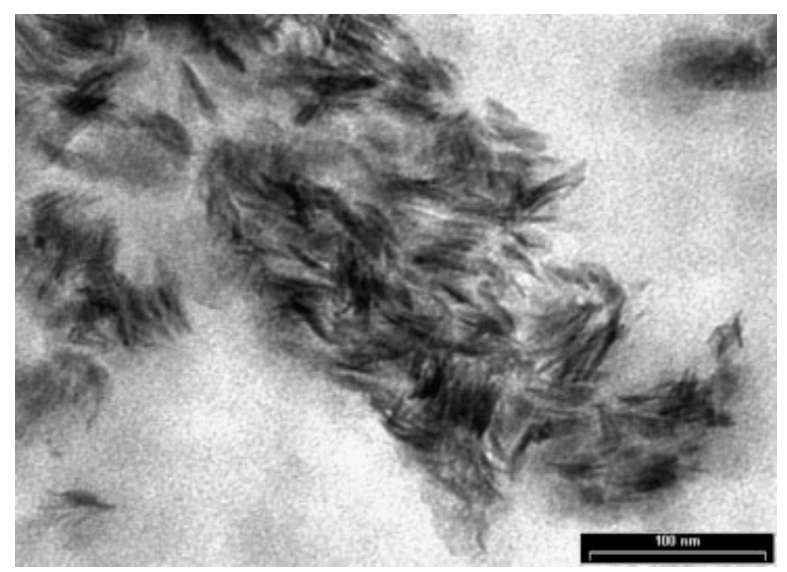

Figure 3. Transmission electron microscopy image of a poly(ether ketone) composite with zirconium phosphate treated with propylammonium and PBI.

of the composite and only assemblies of layers are dispersed in the polymer matrix with the original interlayer distance), (2) the intercalation of the polymer between the layers (i.e., the interlayer distance is increased), and (3) the exfoliation of the layered compound by the polymer (i.e., the layers are no longer parallel).

However, a detailed and semiquantitative evaluation of the composite morphology and distribution of the inorganic component in the polymer matrix could only be achieved by ASAXS. The ASAXS curves are shown in Figure 4.

The composites of phosphates without treatment with $n$-propyl amine and PBI showed a clear diffraction peak at $\mathbf{q}=0.63 \AA^{-1}$ (interlayer distance $=10 \AA$ ). The spacing was similar to that found for the zirconium phosphate with wide-angle X-ray scattering. ${ }^{8}$ A higher interlayer distance (12 A) was observed for the SPEEK/zirconium phosphate composites prepared with treated phosphates, indicating that PBI was kept between the inorganic layers during the casting process. A significant increase in the width of the reflection peak was also observed for this membrane after the intercalation of the polymer. This indicated that the organization of the layers was probably affected by the intercalation process and some extent of exfoliation was initiated.

Furthermore, ASAXS could give additional information about the arrangement of the inorganic layers, taking into account the fractal structure. The bilogarithmic plot of $I(\mathbf{q})$ as a function of $\mathbf{q}$ has a linear segment for both SPEEK/phosphates composites. From the analysis of this linear part of the curve, information about the fractal geom- etry ${ }^{20-23}$ could be obtained according to the theory described in the introduction of this article. Surface-fractal behavior was observed for the SPEEK/phosphate composites without any treatment for inducing exfoliation. Values of $\alpha$ equal to 3.6 and of $D_{\mathrm{s}}$ equal to 2.4 were obtained. The size of the smallest fractal $\left(r_{0}\right)$ unit and the largest fractal aggregate $\left(L_{\mathrm{m}}\right)$ were estimated from the beginning $\left(\mathbf{q}_{\text {min }}\right)$ and end $\left(\mathbf{q}_{\max }\right)$ of the linear segments of the separated SAXS curves according to eq 6 and eq $7:^{41}$

$$
\begin{gathered}
r_{0}=\frac{1}{\mathbf{q}_{\max }} \\
L_{\mathrm{m}}=\frac{\pi}{\mathbf{q}_{\min }}
\end{gathered}
$$
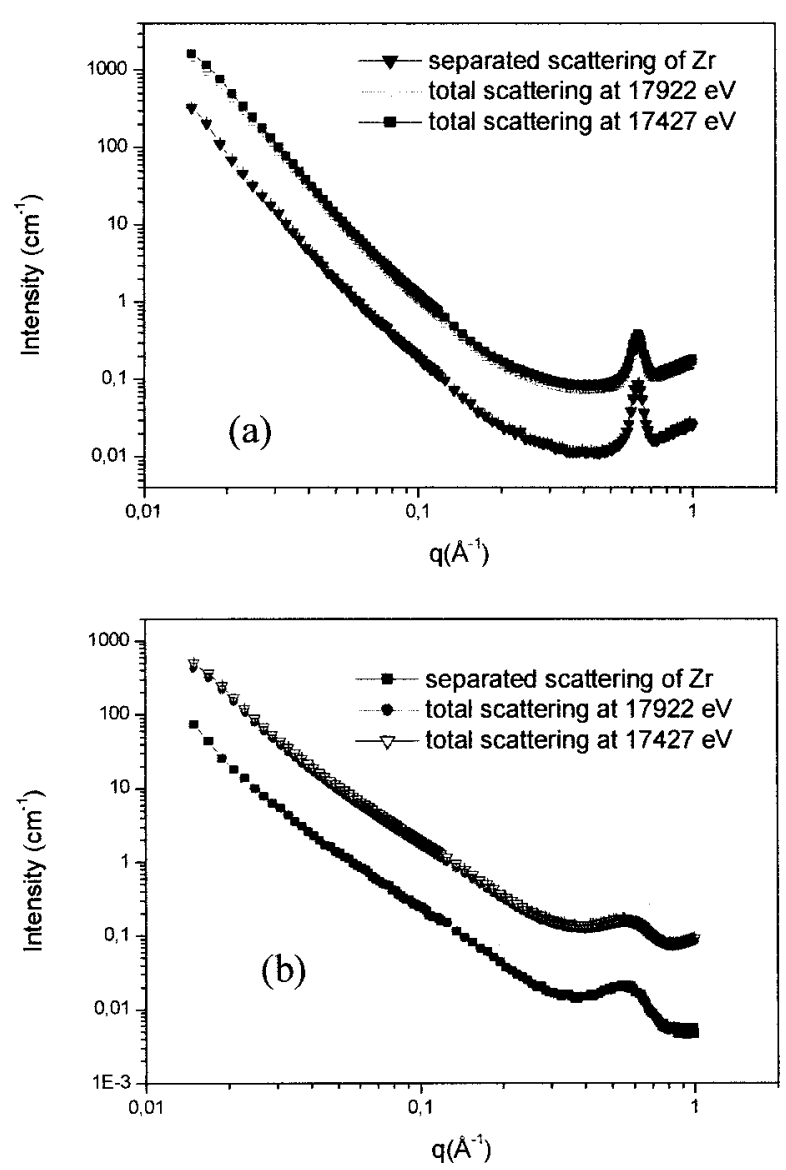

Figure 4. ASAXS plots for SPEEK composites with (a) zirconium phosphates without any pretreatment and (b) zirconium phosphates treated with $n$-propyl amine and PBI, including total and separated scattering curves. 
They correspond to 6.4 and $185 \AA$, respectively. The last value is close to the size of the individual particles revealed by SEM (Fig. 2). The individual particles are the fractal aggregates, with radius $r_{0}$, assembling the small fractal units. These particles are bound together in a fractal structure, which may have radius as big as $L_{\mathrm{m}}$. Moreover, the linear regime, used for the determination of the fractal dimension, was observed in the range $0.017<\mathbf{q}<0.157 \AA^{-1}$, which is far from the diffraction peak, which is located at $\mathbf{q}=0.63$ $\AA^{-1}$.The particles themselves form agglomerates, as shown in Figure 2. Therefore, ASAXS goes further in resolution, giving information about the distribution of fractal structures inside individual particles, which could not be revealed by SEM alone.

A significant difference was observed for the membrane prepared with previously exfoliated zirconium phosphate. In this case, mass-fractal behavior was observed. $D_{\mathrm{v}}$ and $\alpha$ could be determined and were equal to 2.6 and 2.6 , respectively. This clearly indicated that PBI played an important role by separating the zirconium phosphate agglomerates and also the layers themselves because a more open fractal structure was observed for the membrane prepared in the presence of PBI. The limits of the minimum and maximum sizes of the aggregates were also estimated and were equal to 6.3 and $165 \AA$, respectively. Although the fractal behavior of the scattering curves covered only about one order of magnitude in the $\mathbf{q}$ range and was obstructed, especially at a high $\mathbf{q}$ value, by the diffraction peak of the interlayer distance, these values may serve as upper and lower limits of the smallest fractal unit and the largest fractal aggregate, respectively. It must be clarified that the linear behavior was observed in the $\mathbf{q}$ range between 0.019 and 0.314 $\AA^{-1}$; that is, the $\mathbf{q}$ values were much lower than $0.52 \AA^{-1}$ (corresponding to the interlayer distance). Thus, the presence of a diffraction peak did not disturb the determination of the fractal dimension.

\section{CONCLUSIONS}

The microstructure and morphology and other properties (pervaporation and proton conductivity) of the composite SPEEK membranes were strongly dependent on the preparation conditions. Concerning the SPEEK/Z $/ \mathrm{rO}_{2}$ composite membrane, the generation of oxide particles in a poly- mer matrix, with a soluble precursor, allowed a good dispersion of the oxide particles in the composite. This composite could be regarded as a nanocomposite, or even as a molecular composite, because the size of the particles was comparable to that reported for oligomeric zirconium oxo clusters. ${ }^{42}$ The selective contrast, enabled by the ASAXS technique, permitted the investigation of the changes in the microstructure of the SPEEK/ zirconium phosphate membranes in detail. The surface-fractal behavior observed for the membranes containing untreated phosphates suggested that this additive was agglomerated in a dense structure as a result of its low affinity to the polymer matrix, actually forming a microcomposite.

The use of a pretreated phosphate allowed the formation of a nanocomposite, as evidenced by the ASAXS measurements. SEM and ASAXS results showed that the treated phosphate was compatible with SPEEK. This was supported not only by the intercalation/exfoliation process, observed by ASAXS, and by the good dispersion, revealed by SEM micrographs, but also by the mass-fractal behavior observed for this material, identified in the separated scattering curve [see Fig. 4(a)].

The authors thank M. Aderhold for the scanning electron microscopy. L. A. S. A. Prado gives special thanks to Project GKSS 7.T1.00.G.01-HS.3 for its financial support.

\section{REFERENCES AND NOTES}

1. Kickelbick, G. Prog Polym Sci 2003, 28, 83-114.

2. Yu, Y. Y.; Chen, C. Y.; Chen, W. C. Polymer 2003, 44, 593-601.

3. Schubert, U. Chem Mater 2001, 13, 3487-3494.

4. Yoshida, M.; Prasad, P. N. Chem Mater 1996, 8, 235-241.

5. Sforca, M. L.; Yoshida, I. V. P.; Borges, C. P.; Nunes, S. P. J Appl Polym Sci 2001, 82, 178-185.

6. Nunes, S. P.; Peinemann, K. V.; Ohlrogge, K.; Alpers, A.; Keller, M.; Pires, A. T. N. J Membr Sci 1999, 157, 219-226.

7. Nunes, S. P.; Schultz, J.; Peinemann, K. V. J Mater Sci Lett 1996, 15, 1139-1141.

8. Nunes, S. P.; Ruffmann, B.; Rikowski, E.; Vetter, S.; Richau, K. J Membr Sci 2002, 203, 215-225.

9. Redondo, S. U. A.; Radovanovic, E.; Torriani, I. L.; Yoshida, I. V. P. Polymer 2001, 42, 1319-1327.

10. Zoppi, R. A.; das Neves, S.; Nunes, S. P. Polymer 2000, 41, 5461-5470.

11. Prado, L. A. S. A.; Radovanovic, E.; Pastore, H. O.; Yoshida, I. V. P.; Torriani, I. L. J Polym Sci Part A: Polym Chem 2000, 38, 1580-1589. 
12. José, N. M.; Radovanovic, E.; Prado, L. A. S. A.; Yoshida, I. V. P. Acta Microsc 2000, 9, 59-60.

13. Ballauff, M. Curr Opin Colloid Int Sci 2001, 6, 132-139.

14. Jokela, K.; Serimaa, R.; Torkkeli, M.; Etelaniemi, V.; Ekman, K. Chem Mater 2002, 14, 5069-5074.

15. Svergun, D. I.; Shtykova, E. V.; Kozin, M. B.; Volkov, V. V.; Konarev, P. V.; Dembo, A. T.; Shtykova, E. V.; Bronshtein, L. M.; Chernyshov, D. M.; Platonova, O. A.; Yakunin, A. N.; Valetsky, P. M.; Khokhlov, A. R. Crystallogr Rep 2001, 46, 586-595.

16. Torkkeli, M.; Serimaa, R.; Etelaniemi, V.; Toivola, M.; Jokela, K.; Paronen, M.; Sundholm, F. J Polym Sci Part B: Polym Phys 2000, 38, 1734-1748.

17. Register, R. A.; Cooper, S. L. Macromolecules 1990, 23, 310-317.

18. Register, R. A.; Cooper, S. L. Macromolecules 1990 , 23, 318-323.

19. Ding, Y. S.; Hubbard, S. R.; Hodgson, K. O.; Register, R. A.; Cooper, S. L. Macromolecules 1988, 21, 1698-1709.

20. Boukari, H.; Lin, J. S.; Harris, N. T. Chem Mater 1997, 9, 2376-2384.

21. Schaeffer, D. W.; Schmidt, P. Phys Rev Lett 1986, 56, 2199.

22. Bale, H. D.; Keefer, K. D. Phys Rev Lett 1984, 53, 596.

23. Blumen, A.; Schnörer, H. Angew Chem Int Ed Engl 1990, 29, 113-133.

24. Gebel; G.; Loppinet, B. In Ionomer Characterization, Theory and Applications; Schlick, S., Ed.; CRC: Boca Raton, FL, 1996; pp 89-92.

25. Belyakov, V. N.; Linkov, V. M. U.S. Patent 5,932,361, 1999.

26. Alberti, G.; Casciola, M.; Massinelli, L.; Bauer, B. J Membr Sci 2001, 185, 73-81.

27. Dyck, A.; Frisch, D.; Nunes, S. P. J Appl Polym Sci $2002,86,2820-2827$.
28. Haubold, H.-G.; Gruenehagen, K.; Wagener, M.; Jungbluth, H.; Heer, H.; Pfeil, A.; Rongen, H.; Brandenburg, G.; Moeller, R.; Matzerath, J.; Hiller, P.; Hailing, H. Rev Sci Instrum 1989, 60, 19431944.

29. Nguyen, D.; Kim, J. S.; Guiver, M. D.; Eisenberg, A. J Polym Sci Part B: Polym Phys 1999, 37, 32263232.

30. Lorenz, A.; Kickelbick, G.; Schubert, U. Chem Mater 1997, 9, 2551-2560.

31. Tian, D.; Blacher, S.; Jerome, R. Polymer 1999, 40, 951-957.

32. Rhee, S.; White, J. L. J Polym Sci Part B: Polym Phys 2002, 40, 1189-1200.

33. Dahmouche, K.; Santilli, C. V.; Silva, M.; Ribeiro, C. A.; Pulcinelli, S. H.; Craievich, A. F. J Non-Cryst Solids 1999, 247, 108-113.

34. Singhal, A.; Beaucage, G.; Harris, M. T.; Toth, L. M.; Keefer, K. D.; Lin, J. S.; Hu, M. Z. C.; Peterson, J. R. J Non-Cryst Solids 1999, 246, 197-208.

35. Schubert, U.; Trimmel, G.; Moraru, B.; Tesch, W.; Fratzl, P.; Gross, S.; Kickelbick, G.; Hüsing, N. Mater Res Soc Symp Proc 2001, 628, CC2.3.1.

36. Trimmel, G.; Fratzl, P.; Schubert, U. Chem Mater 2000, 12, 602 .

37. Alberti, G.; Casciola, M.; Costantino, U.; Di Gregorio, F. Solid State Ionics 1989, 32, 40-44.

38. Schmidt, D.; Shah, D.; Giannelis, E. P. Curr Opin Solid State Mater Sci 2002, 6, 205-212.

39. Alexandre, M.; Dubois, P. Mater Sci Eng Rep 2000, 28, 1-63.

40. LeBaron, P. C.; Wang, Z.; Pinnavaia, T. J. Appl Clay Sci 1999, 15, 11-29.

41. Schmidt; P. W. In The Fractal Approach to Heterogeneous Chemistry; Avnir, D., Ed.; Wiley: New York, 1989; pp 67-79.

42. Schubert, U. J Sol-Gel Sci Technol 2003, 26, 47-55. 